\title{
Insights into the Relationships Among Capillary Pressure, Saturation, Interfacial Area and Relative Permeability Using Pore-Network Modeling
}

\author{
V. Joekar-Niasar · S. M. Hassanizadeh · A. Leijnse
}

Received: 6 July 2007 / Accepted: 22 November 2007 / Published online: 11 December 2007

(C) The Author(s) 2007

\begin{abstract}
To gain insight in relationships among capillary pressure, interfacial area, saturation, and relative permeability in two-phase flow in porous media, we have developed two types of pore-network models. The first one, called tube model, has only one element type, namely pore throats. The second one is a sphere-and-tube model with both pore bodies and pore throats. We have shown that the two models produce distinctly different curves for capillary pressure and relative permeability. In particular, we find that the tube model cannot reproduce hysteresis. We have investigated some basic issues such as effect of network size, network dimension, and different trapping assumptions in the two networks. We have also obtained curves of fluid-fluid interfacial area versus saturation. We show that the trend of relationship between interfacial area and saturation is largely influenced by trapping assumptions. Through simulating primary and scanning drainage and imbibition cycles, we have generated two surfaces fitted to capillary pressure, saturation, and interfacial area $\left(P_{c}-S_{w}-a_{n w}\right)$ points as well as to relative permeability, saturation, and interfacial area $\left(k_{r}-S_{w}-a_{n w}\right)$ points. The two fitted three-dimensional surfaces show very good correlation with the data points. We have fitted two different surfaces to $P_{c}-S_{w}-a_{n w}$ points for drainage and imbibition separately. The two surfaces do not completely coincide. But, their mean absolute difference decreases with increasing overlap in the statistical distributions of pore bodies and pore throats. We have shown that interfacial area can be considered as an essential variable for diminishing or eliminating the hysteresis observed in capillary pressure-saturation $\left(P_{c}-S_{w}\right)$ and the relative permeability-saturation $\left(k_{r}-S_{w}\right)$ curves.
\end{abstract}

Keywords Capillary pressure $\cdot$ Relative permeability $\cdot$ Pore network model · Interfacial area $\cdot$ Hysteresis

V. Joekar-Niasar · S. M. Hassanizadeh ( $\varangle)$

Environmental Hydrogeology Group, Faculty of Earth Sciences, Utrecht University, Budapestlaan 4, Utrecht 3584 CD, The Netherlands

e-mail: hassanizadeh@geo.uu.nl

A. Leijnse

Soil Physics, Ecohydrology and Groundwater Management Group, Wageningen University,

Droevendaalsesteeg 4, PO Box 47, Wageningen 6700 AA, The Netherlands 


\section{Introduction}

In two-phase systems in porous media, the constitutive equation that relates fluid pressures to saturation plays an important role. Equation (1) states that the difference in fluid pressures, called capillary pressure, is a function of saturation (Bear et al. 1968).

$$
P_{n}-P_{w}=P_{c} \equiv f\left(S_{w}\right),
$$

in which, $P_{n}\left(\mathrm{ML}^{-1} \mathrm{~T}^{-2}\right)$ is the pressure of the nonwetting phase, $P_{w}\left(\mathrm{ML}^{-1} \mathrm{~T}^{-2}\right)$ is the pressure of the wetting phase, $P_{c}\left(\mathrm{ML}^{-1} \mathrm{~T}^{-2}\right)$ is the capillary pressure, and $S_{w}$ is the saturation of the wetting phase. This relationship is known to be hysteretic. For a given soil, many $P_{c}-S_{w}$ curves pertaining to different drainage/imbibition stages and histories are possible. Using a thermodynamically constrained averaging approach, Hassanizadeh and Gray (1990) derived an extended theory of capillarity. According to their results, capillary pressure is not only a function of saturation but also of specific areas of the three interfaces that are present in a two-phase flow system:

$$
P_{c} \equiv f\left(S_{w}, a_{\alpha \beta}\right) ; \quad \alpha \beta=w n, w s, n s
$$

where $a_{\alpha \beta}\left(\mathrm{L}^{-1}\right)$ denotes the specific area of $\alpha \beta$-interface; that is, the area of $\alpha \beta$-interface per unit volume of the porous medium. Here, subscripts $w, n$, and $s$ denote wetting phase, nonwetting phase, and solid phase, respectively. Later, they suggested (Hassanizadeh and Gray 1993a) that the role of $w s$-and $n s$-interfaces are not significant and proposed a simpler relationship:

$$
P_{c} \equiv f\left(S_{w}, a_{n w}\right)
$$

where $a_{n w}$ denotes the specific area of $w n$-interface, which we refer to it simply as "specific interfacial area" in the remainder of this paper.

Equation (3) prescribes a surface relating capillary pressure, saturation and interfacial area. Hassanizadeh and Gray (1993b) conjectured that the resulting surface might be the same for drainage and imbibition, i.e., it might be devoid of hysteresis. This conjecture has been investigated in few studies using computational and experimental approaches (see, e.g., Reeves and Celia 1996; Held and Celia 2001; Chen and Kibbey 2006).

In experimental studies, many researchers have tried to measure the interfacial area under static conditions. These techniques are mostly categorized under two main groups; tracers or imaging techniques. Aqueous tracers have been used by Karkare and Fort (1996), Brusseau et al. (1997), Kim et al. (1997), Saripalli et al. (1998), Anwar (2000), Schaefer et al. (2000), and Chen and Kibbey (2006), and gas tracers have been used in some other studies such as Kim et al. (1999), Costanza-Robinson and Brusseau (2002), and Brusseau et al. (2006). Imaging techniques have been used by Montemagno and Gray (1995), Wildenschild et al. (2002), Cheng et al. (2004), Culligan et al. (2004), Al-Raoush and Willson (2005a,b), and Schnaar and Brusseau (2005, 2006).

Computational approaches have been mainly based on pore-network modeling or latticeBoltzmann models. A valuable tool for the theoretical study of two-phase flow in porous media is pore-network modeling (traditionally called pore-scale network modeling, which is unnecessarily long), introduced for the first time by Fatt (1956). This tool has been used extensively by many researchers for studying various processes in porous media (e.g., Dias and Payatakes 1986; Burganos and Payatakes 1992; Reeves and Celia 1996; Blunt et al. 2002; Gielen 2007). In addition to using pore-network models for theoretical studies, some researchers have tried to develop predictive models for various purposes. Vogel (1997, 2000), 
and Vogel and Roth (1998) used the serial sectioning technique to produce a representative pore-network, and modeled soil relative permeability. Hui and Blunt (2000) have also modeled the relative permeability for a three-phase system using a bundle of tubes of different sizes with constant triangular cross sections. Blunt et al. (2002) have concluded that by combining an appropriate pore-scale physics with a geologically representative description of the pore space, one can produce capillary pressure and relative permeability curves for a given rock without actual measurements. They produced primary drainage and water-flood relative permeabilities for Berea sandstone using pore-network modeling. Piri and Blunt (2005) developed a pore-scale model, which included all important features of immiscible fluid flow at the pore-scale, such as wetting layers, spreading layers of the intermediate-wetting phase, hysteresis, and wettability alteration. The model computes relative permeabilities, saturation paths, and capillary pressure for any displacement sequence. They have reported a good agreement between experiment and simulation results for the relative permeability in a two-phase (water-wet) system.

Despite the wide interest in measuring and/or calculating specific interfacial area and capillary pressure, there are surprisingly very few works on the validity of Eq. 3. There are some experimental studies where $a_{n w}-S_{w}$ curves are produced (see, e.g., Cheng et al. 2004; Chen and Kibbey 2006). These curves are all found to be hysteretic. In the recent experiments by Cheng et al. (2004), $a_{n w}-P_{c}-S_{w}$ surfaces are obtained. They find that the drainage and imbibition surfaces have a difference of only $2.77 \%$.

The first theoretical studies of Eq. 3 were done by Reeves and Celia (1996) and later by Held and Celia (2001) using pore-network models. They developed a static pore-scale cubic lattice network model that included spheres and bi-conical elements representing pore bodies and pore throats, respectively. They did not consider trapping during drainage in their simulations. They could produce smooth and well-behaved three-dimensional surfaces relating saturation, interfacial area, and capillary pressure, based on successive drainage and imbibition cycles. The drainage and imbibition surfaces obtained by Reeves and Celia (1996), however, were distinctly different. So, they concluded that $P_{c}-S_{w}-a_{n w}$ surface was still hysteretic. Moreover, they noticed that the surface was not monotonic; for a given $a_{n w}$ and $S_{w}$, two different values of $P_{c}$ were possible. This led to the proposition to replace Eq. 3 with a relationship for fluid-fluid interfacial area:

$$
a_{n w}=F\left(S_{w}, P_{c}\right)
$$

Later, Held and Celia (2001) showed that the hysteresis in the $a_{n w}-S_{w}-P_{c}$ surface could be almost eliminated if a certain choice of fluid displacement parameters is used. For some specific values for snap-off and local coefficients related to the fluid configuration, they found a separation of less than $1.5 \%$ between imbibition and drainage surfaces. Because, the required displacement rules were in agreement with commonly observed experimental condition, they argued that the modeling of hysteresis in capillary pressure-saturation curves through inclusion of fluid-fluid interfacial area in the formulation of two-phase flow theories is a real possibility. In a recent work, Helland and Skjaeveland (2007) have also studied $a_{n w}-S_{w}-P_{c}$ relationship using mixed-wet triangular bundle of tubes. They have concluded that very different trends in the specific interfacial area vs. saturation curves can occur during imbibition, depending on the reversal point after primary drainage and the advancing contact angle. In addition, they found that hysteresis can be present between imbibition and secondary drainage if contact angle hysteresis is assumed. They also concluded that a more complex model is required if one is to consider effect of phase entrapment and snap-off events.

It is evident that the effect of interfacial area on $S_{w}-P_{c}$ relationship has been studied in very few works. Also, so far no one has studied the role of interfacial area in $k_{r}-S_{w}$ 
relationship. In this study, we focus on understanding the relationships among interfacial area, capillary pressure, saturation, and relative permeability, using pore-network models. We will show that the inclusion of interfacial area in $S_{w}-P_{c}$ and $k_{r}-S_{w}$ relationships may lead to a very significant decrease in the hysteresis observed in these curves. Two different static pore-networks have been developed, and the aforementioned relationships have been studied. One is based on Fatt's model and has only one element type, namely pore throat. The second one is a sphere-and-tube model with both pore bodies and pore throats. We have studied representative elementary volume (REV) size, side boundaries effect, effect of trapping assumptions, and the role of pore size distribution in our models. We investigate the role of interfacial area in qualifying hysteresis observed in $P_{c}-S_{w}$ and $S_{w}-k_{r}$ relationships. To this end, we have produced surfaces linking capillary pressure and saturation to interfacial area $\left(P_{c}-S_{w}-a_{n w}\right.$ surface) as well as saturation and relative permeability to interfacial area $\left(S_{w}-a_{n w}-k_{r}\right.$ surface).

\section{Model Description}

\subsection{Model Structure}

We have developed two types of pore-networks. Both of them have a fixed coordination number of six. The first network is based on Fatt's model (Fatt 1956). It consists of tubes only and is hereafter called the tube network. No volume is assigned to the nodes, where tubes are connected to each other. The second network consists of tubes and spheres, which represent pore throats and pore bodies, respectively. We refer to it as the sphere-and-tube network.

The cross section of network elements is considered to be circular. Radius distribution of pore bodies in the sphere-and-tube network, and radius distribution of pore throats (tubes) in the tube network have been generated using a truncated random log-normal number generator.

In the tube network, after generating the radii of the pore throats, their lengths are determined. We use a relationship between the radius and the length of a pore for sandy soil suggested by Fatt (1956):

$$
l=C r^{-1}
$$

in which, $l$ is the pore length (L), $C$ is an empirical constant $\left(\mathrm{L}^{2}\right)$, and $r$ is the radius of pore (L). It is clear that using this relationship, the ends of the tubes do not necessarily fall on lattice points. In fact, we do not specify how the tubes are geometrically connected to each other.

In the sphere-and-tube network, the length of a pore throat is equal to the spacing of the lattice points minus the sum of radii of the two neighboring pore bodies. The radius of a pore throat is also determined from the radii of neighboring pore bodies. In real porous media, it has been observed that the pore throat radius is correlated with the radii of neighboring pore bodies (see, e.g., Al-Raoush and Willson 2005a,b). Thus, we have formulated a procedure, explained in the Appendix, to determine the radius of a pore throat based on radii of neighboring pore bodies.

The porosity of the network is calculated from the sum of volumes of all pore bodies and pore throats divided by the total volume of the lattice. For fluid displacement simulations, the pore network is considered to be connected at the top to a nonwetting phase reservoir, and at the bottom to a wetting phase reservoir. Side faces are assumed to be no-flow boundaries. 


\subsection{Trapping Assumptions}

For drainage, we have employed two different algorithms regarding the trapping of the wetting phase. In one case, it is assumed that the wetting phase in a pore can be displaced by the nonwetting phase, only if the former is connected to its boundary reservoir. This means when the neighboring pore bodies of a pore throats are filled with the nonwetting phase, that pore throat is considered to be trapped. We refer to this as tight trapping. For example in Fig. 1, if we assume tight trapping, the tube $i j$ will be trapped due to the occupation of the neighboring pore bodies by invading phase. However, in real porous media, it is possible for the wetting phase in a trapped pore throat to escape if at least one of the neighboring pore throats is still connected to the wetting phase reservoir. We refer to this as loose trapping. So, for example, tube $i j$ in Fig. 1 may be still drained into tubes $i l$ and $j k$. If, however, tubes $i l$ and $j k$ are also filled with nonwetting phase, the tubes $i j$ remains trapped. The trapping assumptions can be seen as a consequence of the real geometrical and topological characteristics of voids. With loose trapping assumption, possibility of occurrence of an individual trapped pore element is less than that of the tight trapping cases. Figure 2 schematically shows individual and cluster trapped configurations for loose trapping assumptions.

For imbibition, we have applied the tight trapping assumptions, since the wetting phase intends to fill the corners. When the neighboring pore bodies of a pore throats are filled completely with the wetting phase, that pore throat is considered to be trapped.

In our simulations of imbibition, no snap-off has been considered. Mahmud and Nguyen (2006) have shown in a recent study that the occurrence frequency of piston-like movement of the wetting phase for contact angles larger than 30 degrees in a spatially uncorrelated network is about twice that of snap-off movements. According to their conclusion, if there is no spatial correlation between the pores (similar to our pore-network model), the role of

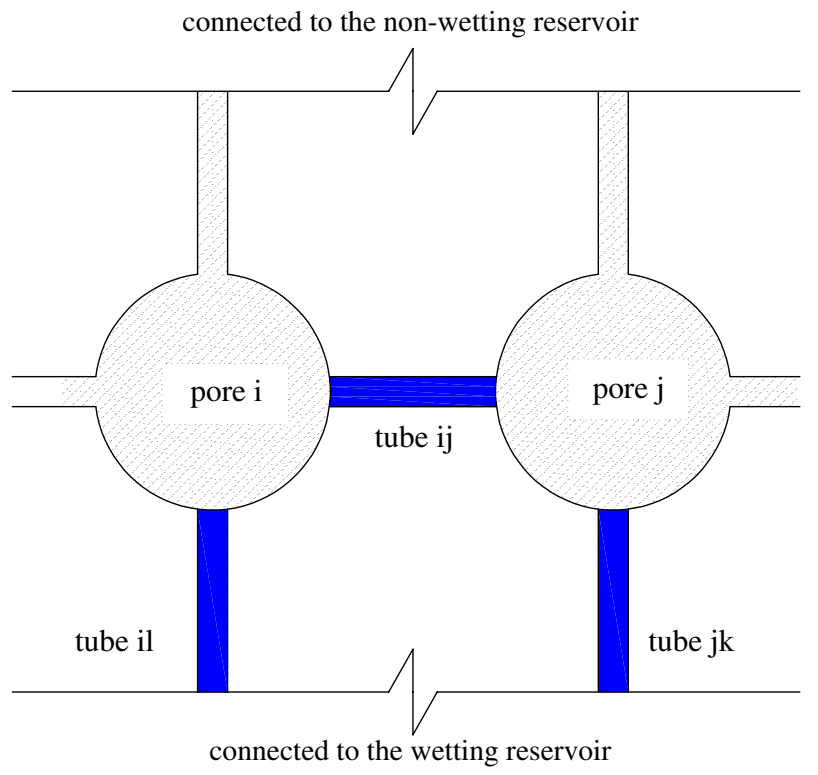

Fig. 1 Schematic illustration of the trapping assumptions during drainage 


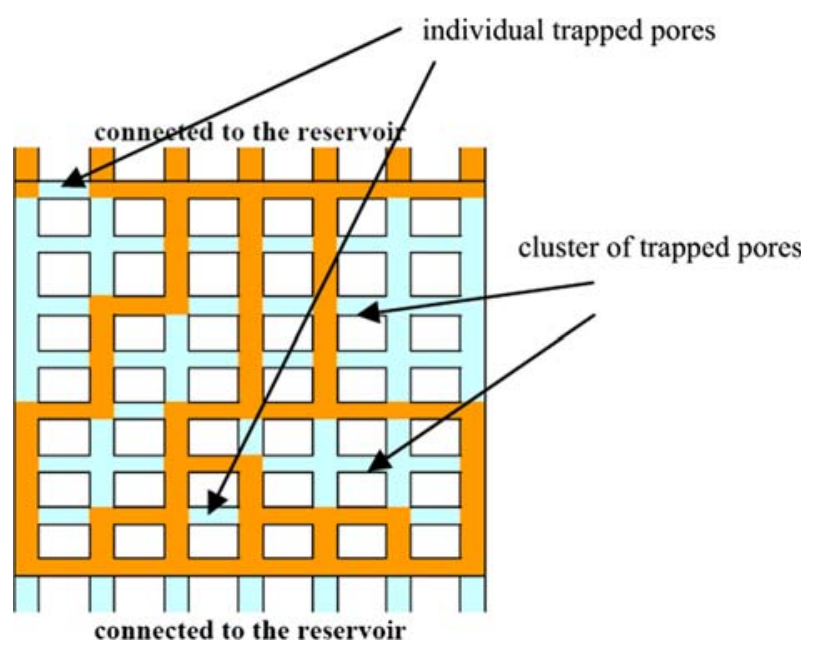

Fig. 2 Schematic presentation of individual and cluster trapped pore

snap-off movements in fluid configuration is not important. Thus, we have not considered the snap-off mechanism in our simulations.

\section{Numerical Experiments}

Conventional drainage and imbibition experiments have been simulated with the two pore networks. Primary and/or main drainage and imbibition curves as well as scanning curves are obtained following the procedure described farther below. For an imposed capillary pressure, the occupancy of pore throats and pore bodies, under imbibition or drainage and assuming equilibrium, has to be determined. To do so, we need to have the entry capillary pressure for a pore throat, which is calculated using Young-Laplace equation:

$$
P_{c}=P_{n}-P_{w}=\frac{2 \sigma \cos \theta}{r},
$$

where $\sigma$ is the interfacial tension $\left(\mathrm{MT}^{-2}\right), r$ is the radius of pore $(\mathrm{L})$, and $\theta$ is the contact angle between solid surface and fluid-fluid interface. The entry pressure of a pore throat in our network is always larger than that of its neighboring pore bodies, because it has a smaller radius than the connected pore bodies.

At any imposed capillary pressure, saturation of each phase can be easily calculated as we know the occupancy of the network. We can also calculate the interfacial areas as we know the location of fluid-fluid interfaces for any given configuration of fluids. During drainage, interface area is calculated based on the radius of the pore throats where the interface is located. However, during imbibition, we may also interface in the pore bodies. The interface area in pore bodies is calculated based on the curvature of interface, which can be obtained from Eq. 6, for an imposed capillary pressure. As shown in Fig. 3, we have not considered existence of multiple interfaces in a pore body even if the wetting phase enters a pore body through two or more pore throat. Area of the interface is calculated based on the curvature assigned by the imposed capillary pressure. Thus, at each capillary pressure, corresponding wetting (or nonwetting) saturation and interfacial area will be known. So, our simulations result in a large 




Fig. 3 Schematic of interface positioning during imbibition. ' $R$ ' is radius of pore body, ' $r$ ' is radius of interface dictated by the imposed capillary pressure, and ' $\theta$ ' is the contact angle. The pore body will be filled up gradually controlled by ' $r$ '. As soon as ' $r$ ' is equal to ' $R$ ', the pore body will be filled up instantaneously

number of $P_{c}-S_{w}-a_{n w}$ data points. Using these data, and producing $P_{c}-S_{w}-a_{n w}$ surfaces for drainage and imbibition, we can investigate the role of interfacial area in eliminating the hysteresis observed in $P_{c}-S_{w}$ curve.

\subsection{Drainage Steps}

Initially, the network is assumed to be saturated with the wetting phase. The pressure of the wetting phase reservoir is assumed to be zero and not changing. Therefore, the pressure of wetting phase in all pores connected to the wetting phase reservoir is also zero until a pore becomes trapped. Initially, the pressure of nonwetting phase reservoir is zero, and thus the imposed capillary pressure is also zero. Primary drainage simulations start by increasing the pressure of nonwetting phase reservoir until it exceeds the entry pressure of the largest pore throat connected to the reservoir. As the radius of a pore body is larger than that of a pore throat, the controlling element is the pore throat. Thus, as soon as the pressure is high enough to enter a pore throat, nonwetting phase will occupy that pore and the connected pore body. When no other pores can be occupied in that pressure step, the nonwetting phase pressure will be increased and more pores will be occupied by the nonwetting phase. Drainage continues, with incrementally increasing nonwetting phase pressure, until the last row of pore throats is filled by the nonwetting phase. Scanning drainage curves are obtained following imbibition simulations by reversing the imbibition process (described below) at various saturations.

\subsection{Imbibition Steps}

Imbibition is simulated by decreasing the pressure of nonwetting phase reservoir (or increasing the pressure of wetting phase reservoir), causing the nonwetting phase to recede. The replacement of the nonwetting phase by the wetting phase starts from those pore throats that have the highest entry pressure (smallest size). Then, as shown in Fig. 3, the wetting phase enters the neighboring pore body and stops at a position with a curvature corresponding to 
the imposed capillary pressure. In each pore body, one interface is permitted, regardless the occupancy of the connected pore throats. As the capillary pressure is decreased, the wetting phase gradually fills the pore body. As soon as the interface radius is equal to the pore body radius, the rest of the pore body and the connected pore throats will be filled up instantaneously by the wetting phase. Hence, the governing element during imbibition is the pore body.

\subsection{Relative Permeability}

Relative permeability for each phase is commonly determined as a function of average saturation. The relationship is known to be hysteretic. We would like to establish whether wettingnonwetting interfacial area plays a role in this relationship. In this section, we describe how relative permeability is determined for a succession of different fluids configurations in a network, corresponding to the full range of saturation.

As described above, in simulations of drainage and imbibition at any imposed $P_{c}$, we obtain a static fluids configuration in the network. For each configuration, we calculate the relative permeabilities for the two phases. This will be done by assigning a pressure difference within each phase across the network, and calculating flow rate and consequently relative permeability for each phase.

The flow rate in each pore throat is calculated using the Poiseuille's formula:

$$
q_{i j}=\frac{\pi}{8 \mu} r_{i j}^{4} \frac{P_{i}-P_{j}}{l_{i j}}
$$

in which $q_{i j}$ is the discharge through the tube from pore body $i$ to $j, \mu$ is the viscosity, $P_{i}$ and $P_{j}$ are the pressures in pore bodies $i$ and $j$, respectively, and $l_{i j}$ is the length of the pore throat. The volume balance for each pore body $i$ would require:

$$
\sum_{j=1}^{N_{i}} q_{i j}=\frac{\pi}{8 \mu} \sum_{j=1}^{N_{i}} r_{i j}^{4} \frac{P_{i}-P_{j}}{l_{i j}}=0
$$

Writing this equation for each pore body results in a set of linear equations, $\mathbf{A} x=b$, which can be solved for the pressure in each fluid phase. In matrix $\mathbf{A}$, for a given phase, only those pore bodies are involved that are connected to both upstream and downstream reservoirs, as there is no flow in dead-end or isolated pores. Having determined the pressure field in the network, the total flow rate through the network for each phase can be calculated.

\section{Results and Discussion}

\subsection{REV in Pore-Network Model}

One of the first questions we investigated was the minimum network size for which the results become insensitive to the network size. This representative network size may be considered to correspond to the REV for the porous medium being modeled. The REV size of the network has been determined based on the value of irreducible saturation as well as $S_{w}-a_{n w}$ relationship. Determination of REV is also essential for testing the validity of results for a given statistical distribution and for saving computer time and memory, as we do not want to work with a too large network. 
Figure 4a shows values of irreducible wetting phase saturation for different sizes of network. It confirms that irreducible saturation does not change significantly for cubic networks larger than 40 nodes in each coordinate direction. Similarly, Fig. 4b shows that for a specific statistical distribution of pore elements, the slope of $S_{w}-a_{n w}$ curve is not significantly influenced by the cubic network size larger than 40 nodes.

We are also interested to see what the effect of no-flow side boundaries on the results is. This is done by varying the size of the network cross section perpendicular to the flow, and comparing resulting $P_{c}-S_{w}$ and $S_{w}-a_{n w}$ curves of sphere-and-tube network and tube network, given in Fig. 5a and b, respectively. As it is illustrated, results of networks with cross sections of $10 \times 40$ nodes and $40 \times 40$ nodes are almost identical. Thus, we conclude that there is no effect from assuming no-flow side boundaries of the network.

However, as expected, there is a significant difference between the results of twodimensional (e.g., $1 \times 40 \times 40$ ) networks and three-dimensional networks (e.g., $2 \times 40 \times 40$ or larger). This is mainly due to the differences in the coordination number. Coordination number for internal nodes of the $1 \times 40 \times 40$ network is 4 , for $2 \times 40 \times 40$ network is 5 , and for larger networks is 6 . A smaller coordination number means less possibility for escape of the receding phase, and this influences the results significantly.

\subsection{Effect of Trapping Assumptions}

As shown in Fig. 6a, variation of interfacial area with saturation can be influenced by different trapping assumptions we make in the simulations. Figure 6a shows the variation of interfacial


Fig. 4 REV definition for networks in terms of irreducible saturation and $a_{n} w$ curve. (a) Irreducible saturation vs. size of network. (b) Specific $a_{n w}$ vs. $S_{w}$ during drainage for tube and sphere-and-tube networks with different sizes of 40,20 , and 10 nodes
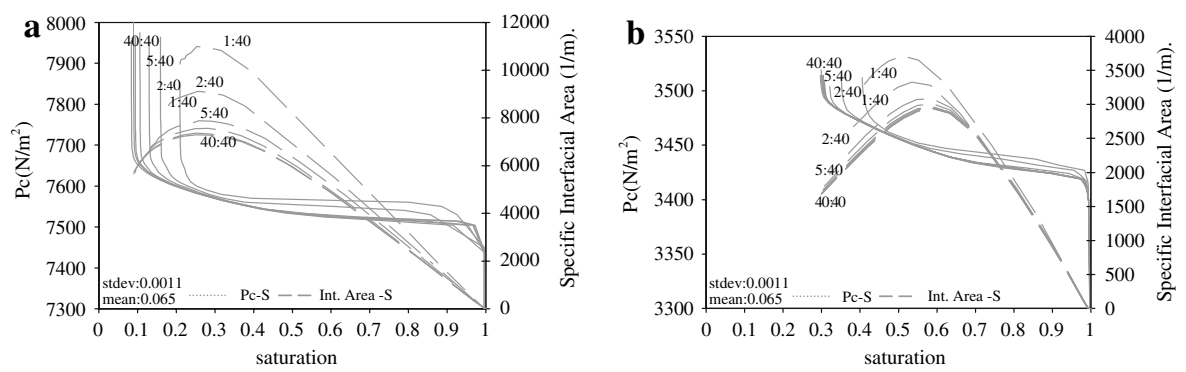

Fig. 5 Effect of boundary on $P_{c}-S_{w}$ and $S_{w}-a_{n w}$ curves for drainage. (a) Sphere-and-tube network. (b) Tube network. Dashed lines show $S_{w}-a_{n w}$ relationship, and solid lines show $P_{c}-S_{w}$ relationship. The full size network is a cubic 40-node network. The curves are shown for different cross section sizes normal to the flow direction 



Fig. 6 Qualitative comparison between the model results and experiments for $a_{n w}-S_{w}$ relationship. (a) Variation of interfacial area with saturation under drainage for three different trapping assumptions. Solid black line shows tight trapping, solid gray line shows loose trapping and dashed gray line shows no trapping. (b) Graph of interfacial area vs. saturation obtained by microtomography on glass beads (Culligan et al. 2006)

area with saturation under three different assumptions; no trapping, loose trapping and tight trapping. Drainage simulations with these three assumptions are done up to the irreducible saturation. Under the tight trapping assumption, interfacial area increases monotonically with decreasing saturation during drainage, while in no-trapping or loose trapping conditions it reaches a maximum value. In the saturation range of $100 \%$ to $55-60 \%$, all curves are almost the same. As the invasion of nonwetting fluid into the porous medium occurs, more and more interfacial area is created until the clusters of invading fluid reconnect. At that stage, in some parts of the network, not only no new interfacial area is created, but also interfacial area disappears due to the re-connection of invading clusters. The tight trapping and no-trapping curves show the extremes of $a_{n w}-S$ curves for various trapping assumptions. Figure 6a illustrates that the trapping conditions can control the irreducible saturation to be $0 \%, 15 \%$, or 30\% for no-trapping, loose-trapping, or tight-trapping conditions, respectively.

Qualitative comparison between our results and experiments on real porous media (Fig. 6b) suggests that loose trapping assumption in modeling seems more in agreement with some real porous media. In addition, the order of magnitude of specific interfacial area in simulations is in agreement with measurements done using imaging techniques in real porous media (e.g., Brusseau et al. 2006; Culligan et al. 2004, 2006).

\section{$4.3 P_{c}-S_{w}$ and $S_{w}-a_{n w}$ relationships}

Figures 7 and 8 show $P_{c}-S_{w}$ and $S_{w}-a_{n w}$ curves, respectively, from a series of networks with different pore size distributions. These curves have been obtained from tube and sphereand-tube networks with loose trapping assumption. Log-normal distributions of pore body radii with mean and standard deviation of $0.065 \mathrm{~mm}$ and $0.02 \mathrm{~mm}^{2}$, respectively, have been employed. In each case, curves for 20 realizations for uncorrelated networks as well as the average curve with cubic size of either 20 or 40 nodes are shown. With increasing network size, band width of variation of $P_{c}-S_{w}$ and $S_{w}-a_{n w}$ curves decreases. This implies that to obtain the average $P_{c}-S_{w}$ and $S_{w}-a_{n w}$ curves of a specific pore-network model, with increase of network size, fewer realizations will be required. Furthermore, our simulations showed (not presented here) that for larger variances more realizations are required to achieve the average behavior.

As seen in Fig. 7, the two types of networks produce different $P_{c}-S_{w}$ curves. This is partly due to the fact that we assign the same size distribution to pore bodies in the sphere-and-tube 



Fig. $7 \quad P_{c}-S_{w}$ curves for drainage with loose trapping assumption. Thin and thick solid lines show realizations and the average curve, respectively
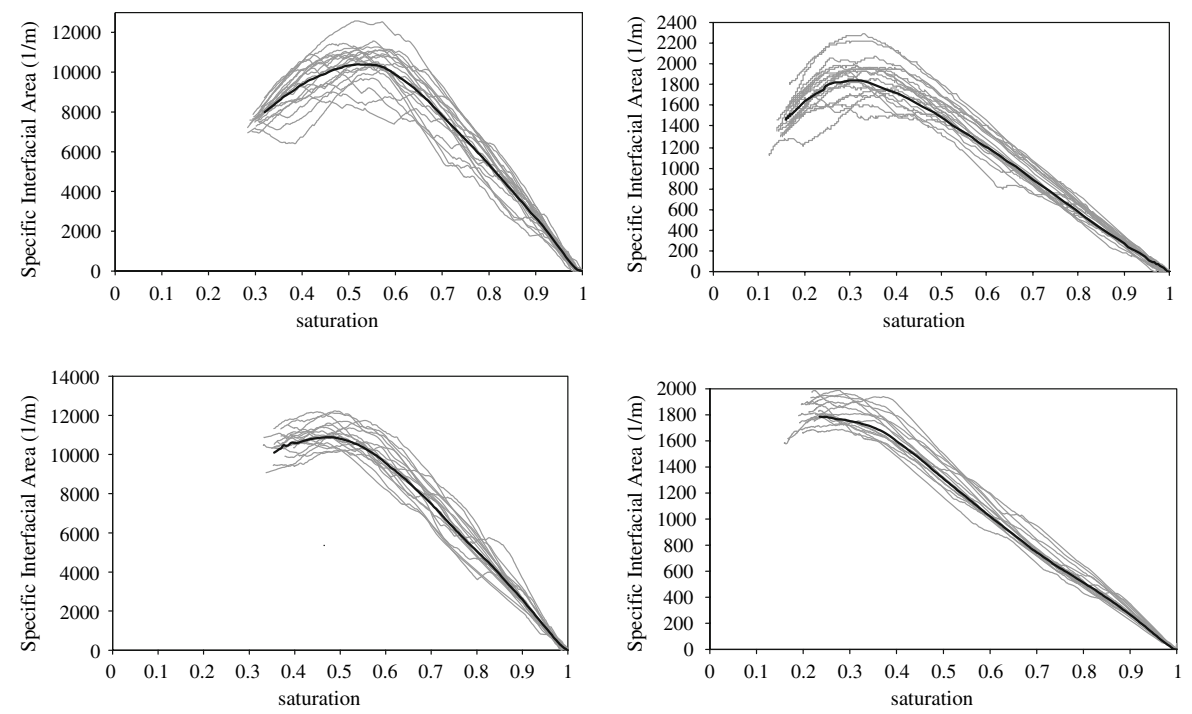

Fig. $8 S_{w}-a_{n w}$ curves for drainage with loose trapping assumption. Thin solid and thick lines show realizations and the average curve, respectively

network and to pore throats in the tube network. Thus, the minimum size of the pore throats in the tube network is the same as the minimum pore body size in the sphere-and-tube network. The latter is always larger than the minimum pore throat size in that network. That procedure sometimes results in very small pore throats. As a result, the range of capillary pressure and interfacial area in the two networks are quite different. 

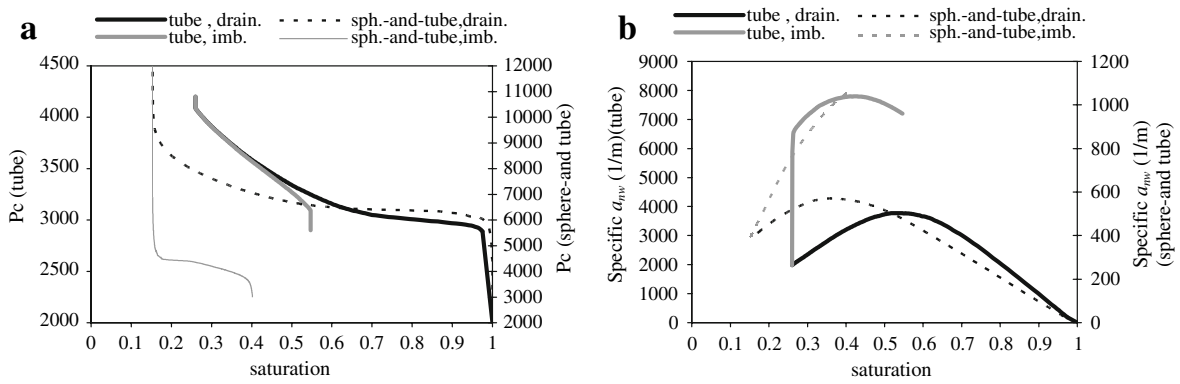

Fig. 9 Typical $P_{c}-S_{w}$ and $S_{w}-a_{n w}$ curves of drainage and imbibition for tube and sphere-and-tube networks. (a) $P_{c}-S_{w}$ relationship. (b) $S_{w}-a_{n w}$ relationship. Solid lines are related to the tube network and dashed lines show sphere-and-tube network results. Black lines represent drainage, and gray lines represent imbibition

Figure 9a shows that, as there is only one network element (namely pore throats) in the tube model, it cannot reproduce hysteresis in a $P_{c}-S_{w}$ curve. Furthermore, as shown in Fig. 9b, there is a jump in interfacial area at the beginning of imbibition experiment in the tube network. Since there is no pore body, there is no gradual change of interfacial area during imbibition, contrary to the sphere-and-tube network results. As seen in Fig. 9a, when the controlling elements during drainage and imbibition are different (as in the sphere-and-tube network), hysteresis in $P_{c}-S_{w}$ curves can be produced.

In recent experiments for determining the $S_{w}-a_{n w}$ relationship (e.g., Culligan et al. 2004, 2006), it was found that the maximum interfacial area occurs at saturations around $30 \%$. Figures 8 and 9 show that this maximum value occurs at saturation of $25-30 \%$ in the sphereand-tube and at saturation of $45-55 \%$ in the tube network. It is evident that the behavior of the sphere-and-tube model is more realistic in representing the soil characteristics than the tube model. Although this result may have been expected, it is important to have it established with the aid of model simulation.

\subsection{Capillary Pressure, Saturation, and Interfacial Area $\left(P_{c}-S_{w}-a_{n w}\right)$ Surface}

Using data points from primary and scanning curves of drainage and imbibition simulations in sphere-and-tube network, we can produce a surface relating capillary pressure, saturation and interfacial area. The full set of drainage and imbibition curves are shown in Fig. 10a and $\mathrm{b}$, respectively. The corresponding interfacial area points are plotted in Fig. 10c. We were able to fit a second-order polynomial surface to the data points. As shown in Table 1, there is a very good correlation coefficient $(0.956)$ for the fitted surface. We have also fitted two separate surfaces to $P_{c}-S_{w}-a_{n w}$ drainage and imbibition data points as shown in Fig. 11. The mean absolute difference between these two surfaces is $8.5 \%$. We also produced such surfaces for a pore network with disconnected statistical distributions for pore bodies and pore throats. As usual, the pore throats are smaller than pore bodies and their distributions have no overlap. In this case (results are not presented here), the mean absolute difference between the two surfaces was much larger (about 18\%). These results show that if the size distributions of pore bodies and pore throat are correlated and show overlap, one may expect less difference between imbibition and drainage surfaces. As Al-Raoush and Willson (2005a,b) have shown in their experimental measurements, the size distributions of pore bodies and pore throats do have a large overlap and it is hard to distinguish pore bodies from pore throats in real porous media. Thus we expect less difference between imbibition and drainage surfaces in 

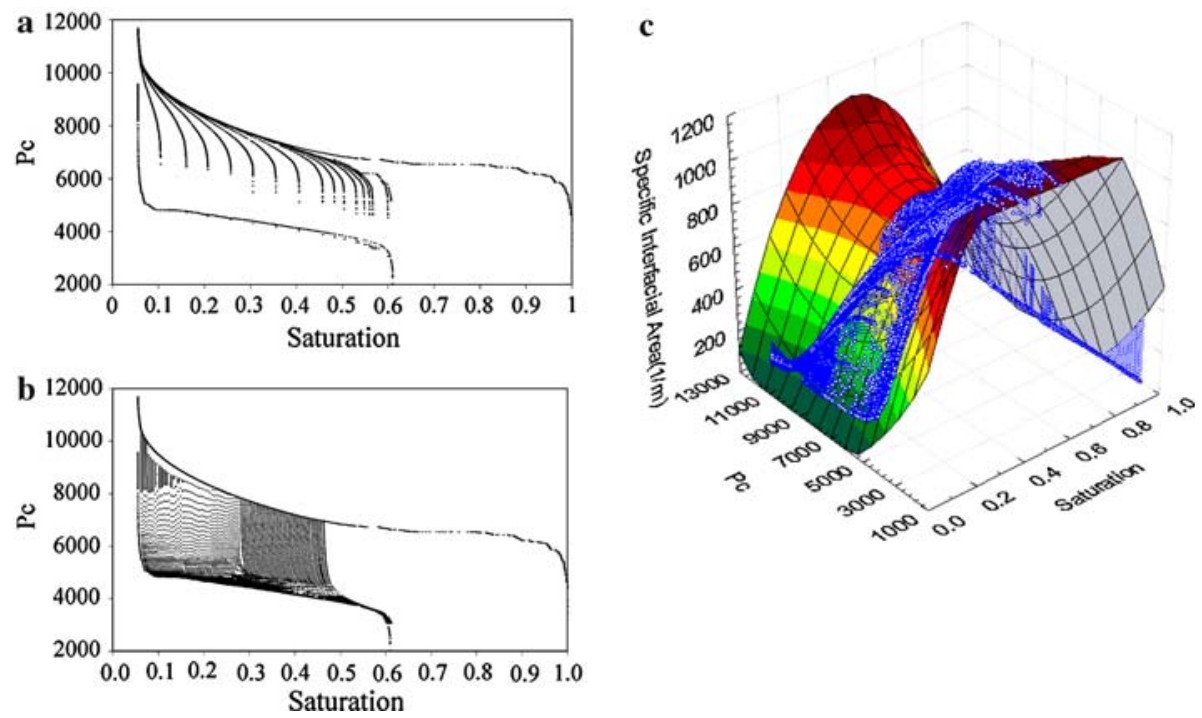

Fig. 10 Relationship among $P_{c}\left(\mathrm{~N} / \mathrm{m}^{2}\right), S_{w}$, and $a_{n w}(1 / \mathrm{m})$ for (a) Drainage curves, (b) imbibition curves, and (c) all $P_{c}-S_{w}-a_{n w}$ points with fitted second-order polynomial surface

Table 1 Statistical properties of the model results and fitted surface

\begin{tabular}{|c|c|c|c|c|}
\hline \multirow[t]{2}{*}{ Item } & \multicolumn{2}{|c|}{$\begin{array}{l}k_{r w}-S_{w}-a_{n w} \text { surface } \\
R^{2}=0.991\end{array}$} & \multicolumn{2}{|c|}{$\begin{array}{l}P_{c}-S_{w}-a_{n w} \text { surface } \\
R^{2}=0.956\end{array}$} \\
\hline & Model results & Fitted surface & Model results & Fitted surface \\
\hline No. of data points & 566 & 566 & 11,887 & 11,887 \\
\hline Mean & 0.16 & 0.158 & 534.0 & 529.9 \\
\hline SD & 0.163 & 0.162 & 346 & 343 \\
\hline
\end{tabular}

correlated pore-network models mimicking the reality, as compared to uncorrelated porenetwork models.

\subsection{Relative Permeability, Saturation, and Interfacial Area $\left(k_{r w}-S_{w}-a_{n w}\right)$ Surface}

After calculating relative permeability at various average saturations in the sphere-and-tube network, we can construct $k_{r w}-S_{w}-a_{n w}$ surface similar to the work done by Reeves (1997). Figure 12a shows relative permeability-saturation curves for the two phases during drainage and imbibition. It shows that the hysteresis observed in the $k_{r w}-S_{w}$ relationship is not as strong as the hysteresis observed in $P_{c}-S_{w}$ relationship. Figure $12 \mathrm{~b}$ shows a typical $k_{r}-a_{n w}$ relationship for the two phases. It can be observed that at a given interfacial area, two different values for the relative permeability are possible, and hysteresis observed in $k_{r}-a_{n w}$ relationship is more significant than that of $k_{r}-S_{w}$ relationship. Finally, a three-dimensional representation of the $k_{r w}-S_{w}-a_{n w}$ surface for the wetting phase is shown in Fig. 12c.

We have fitted a second-order polynomial surface to $P_{c}-S_{w}-a_{n w}$ and $k_{r w}-S_{w}-a_{n w}$ simulation data of the sphere-and-tube network with loose trapping assumption. Statistical prop- 

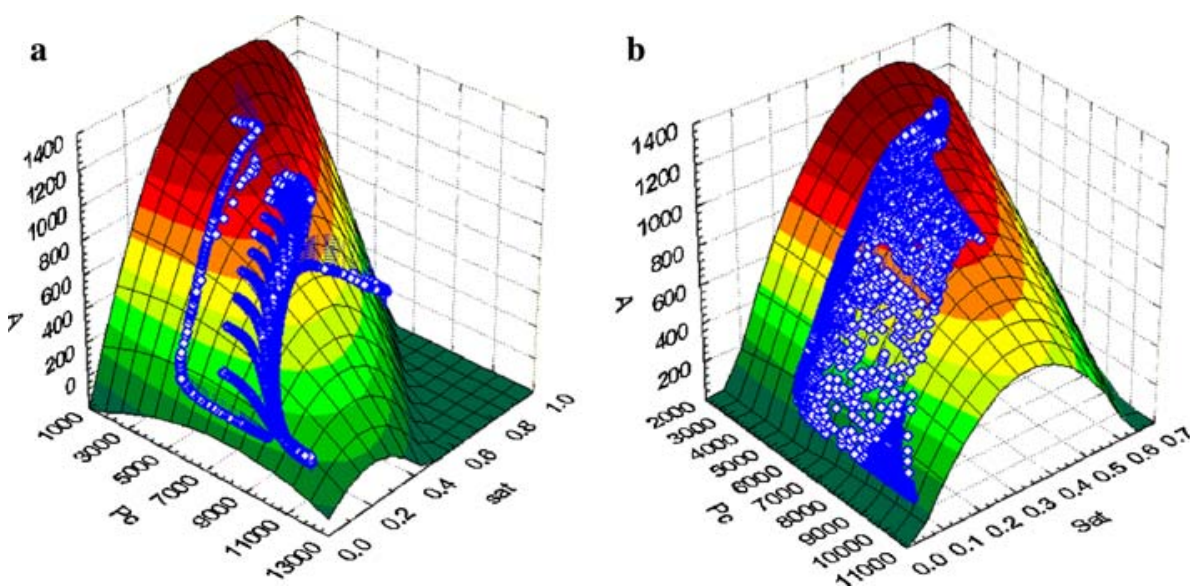

Fig. 11 (a) $P_{c}-S_{w}-a_{n w}$ drainage data points with fitted second-order polynomial surface. (b) $P_{c}-S_{w}-a_{n w}$ imbibition data points with fitted second-order polynomial surface


Fig. 12 Relationship among $k_{r w}, S_{w}$ and $a_{n w}$. Solid lines correspond to the nonwetting phase and dashed lines correspond to the wetting phase. (a) Main and scanning $k_{r w}-S_{w}$ curves, (b) Main and scanning $k_{r w}-a_{n w}$ curves, and (c) the fitted $k_{r w}-S_{w}-a_{n w}$ surface with polynomial equation

erties of the model results and fitted surfaces are shown in Table 1. We see that the correlation coefficients are very high. We also found that the maximum deviation happens during the primary drainage at high saturations. The equations of fitted surfaces are as follows (in SI unit): 


$$
\begin{aligned}
a_{n w}= & 849+3858 S_{w}-0.224 P_{c}-3992 S_{w}^{2}+0.006 S_{w} \cdot P_{c}+(1.283 \mathrm{E}-5) P_{c}^{2} \\
k_{r w}= & 1.26 S_{w}-(4.39 \mathrm{E}-4) a_{n w}-0.32 S_{w}^{2}+(2.301 \mathrm{E}-4) S_{w} \cdot a_{n w} \\
& +(1.447 \mathrm{E}-7) a_{n w}^{2}
\end{aligned}
$$

\section{Summary and Conclusions}

We have studied relationships among capillary pressure, saturation, and interfacial area $\left(P_{c}-S_{w}-a_{n w}\right)$ as well as relative permeability, saturation, and interfacial area $\left(k_{r w}-S_{w}-a_{n w}\right)$. To understand how network modeling algorithms influence the results, two different porenetwork models have been developed; a tube network (similar to Fatt's model) which has no pore bodies, and a sphere-and-tube network with both pore throats and pore bodies.

Our results show that the oversimplification of the network influences the results significantly. In general, the sphere-and-tube network produces more realistic results than the tube network. The tube network is unable to produce hysteresis. Considering hysteresis in $P_{c}-S_{w}$ relationship as a basis for resemblance of pore networks to the real porous media, we can conclude that the two network elements, namely pore throats and pore bodies, are required to produce hysteresis.

Based on the irreducible saturation for an uncorrelated pore size distribution with sphereand-tube lattice geometry, we need about 40 nodes in each direction in order to have a representative network (REV). With an ensemble of 20 realizations, we could produce representative $P_{c}-S_{w}-a_{n w}$ curves. The required size of ensemble decreases with increasing the network size.

Two different trapping algorithms have been employed: loose trapping (which mimics the escaping of wetting phase via corners of pores) and tight trapping (which assumes that the wetting phase trapped in a pore cannot escape). Trapping assumptions can influence significantly the form of $S_{w}-a_{n w}$ curves as well as irreducible saturation. However, the trapping assumption is not important in wetting saturations larger than $55 \%$. For the case of tight trapping, interfacial area increases monotonically with decreasing saturation. But, for the case of loose trapping, the variation is not monotonic and a maximum interfacial area can be observed at saturations around $25-35 \%$, similar to the experiments on the real porous media. It implies that loose trapping assumption is more in agreement with real conditions.

Using data from the full range of scanning drainage and imbibition simulations, we have constructed $P_{c}-S_{w}-a_{n w}$ and $k_{r w}-S_{w}-a_{n w}$ surfaces. We have fitted a second-order polynomial to these surfaces, with correlation coefficients of 0.991 and 0.956 , respectively. Furthermore, we have fitted a surface to the $P_{c}-S_{w}-a_{n w}$ data points for drainage and imbibition, separately. These surfaces have been produced for two networks with different values of statistical distributions of pore bodies and pore throats. Results show that with increasing the overlapping part of the pore body and pore throat size distributions, the absolute difference between the two surfaces decreases. This implies that in a real porous medium, where there is no clear distinction between pore bodies and pore throats, we can expect a smaller difference between the surfaces. Results of relative permeability-interfacial area show that there is a stronger hysteresis in $k_{r w}-a_{n w}$ curves than in $k_{r w}-S_{w}$ curves.

Results of this work suggest that in multiphase systems, interfacial area can be an essential variable in $P_{c}-S_{w}$ and $k_{r w}-S_{w}$ relationships. It can help in diminishing or eliminating the hysteresis that is commonly observed in $P_{c}-S_{w}$ and $k_{r w}-S_{w}$ curves.

Acknowledgements The authors are grateful to the anonymous reviewers for their valuable comments. 
Open Access This article is distributed under the terms of the Creative Commons Attribution Noncommercial License which permits any noncommercial use, distribution, and reproduction in any medium, provided the original author(s) and source are credited.

\section{Appendix: Computation of Pore Throat Radii}

For our sphere-and-tube network, we specify a size distribution for the pore bodies. Then, the radius of a pore throat is determined from the radii of the two neighboring pore bodies. We do this following a scheme adopted from Acharya et al. (2004) and explained below.

Consider two pore bodies $i$ and $j$, with a center-to-center distance " $d$ " (see Fig. A.a), and pore sizes $R_{i}$ and $R_{j}$, respectively. Along the line connecting the centers of the two nodes, we can define the dimensionless coordinate $\xi$ :

$$
\xi=x / d, \quad 0 \leq \xi \leq 1
$$

We consider two arbitrary curves with order of $n>0$, tangent to the pore bodies (see Fig. A) with the following equations:

$$
\begin{gathered}
\rho_{1}(\xi)=\rho_{i}(1-\xi)^{n} \quad \text { for } 0 \leq \xi \leq 1 \\
\rho_{2}(\xi)=\rho_{j} \xi^{n} \text { for } 0 \leq \xi \leq 1
\end{gathered}
$$

where parameters $\rho_{i}$ and $\rho_{j}$ (see Fig. A.b) need to be determined following the procedure explained below.

The points of contact of these curves with the pore bodies $i$ and $j$ are denoted by $a$ and $c$, respectively. The points $a$ and $c$ are determined so that the angle between the radii passing through these points and the horizontal axis is $\pi / 4$. The two curves intersect each other at point $b$. The projections of these points on the dimensionless coordinate axis $\xi$ are denoted by $\tilde{a}, \tilde{b}$ and $\tilde{c}$ (see Fig. A.1.b). At point $b$, we have $\rho_{1}(\tilde{b})=\rho_{2}(\tilde{b})$. So using Eqs. A.2 and
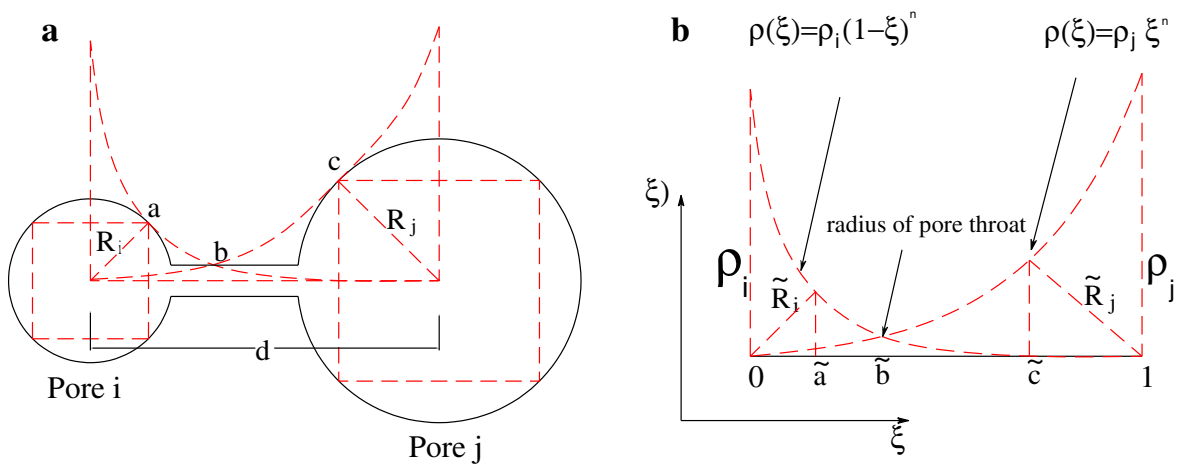

Fig. A Geometrical configuration of relationship between pore body and pore throat radii in sphere-and-tube network. (a) Geometrical configuration for determining the pore throat radius. (b) Schematic definition of functions used for determining the radius of pore throat. Radius of pore throat is determined at intersection of the two curves (point b) using the dimensionless configuration in (b) 
A.3, we can determine $\tilde{b}$ :

$$
\rho_{i}(1-\tilde{b})^{n}=\rho_{j} \tilde{b}^{n} \rightarrow \tilde{b}=\frac{\left(\rho_{i} / \rho_{j}\right)^{1 / n}}{1+\left(\rho_{i} / \rho_{j}\right)^{1 / n}}
$$

We now need to calculate $\rho_{\alpha}(\alpha=i, j)$. From geometrical considerations, we can write for pore body $i$ :

$$
\tilde{a}=\tilde{R}_{i} \cos (\pi / 4), \quad \rho_{1}(\tilde{a})=\tilde{R}_{i} \sin (\pi / 4),
$$

where $\tilde{R}_{\alpha}=R_{\alpha} / d$ is the dimensionless radius.

In addition, from Eq. A.3, we can write:

$$
\rho_{1}(\tilde{a})=\rho_{i}(1-\tilde{a})^{n}
$$

Substituting (A.5) in (A.6), we can calculate $\rho_{i}$ :

$$
\rho_{i}=\frac{\tilde{R}_{i} \sin (\pi / 4)}{\left(1-\tilde{R}_{i} \cos (\pi / 4)\right)^{n}}
$$

In a similar fashion, $\rho_{j}$ is calculated:

$$
\rho_{j}=\tilde{R}_{j} \sin (\pi / 4) /\left(1-\tilde{R}_{j} \cos (\pi / 4)\right)^{n}
$$

Combining Eqs. A.8, A.3, and A.4, the radius at the intersection point (b), which is taken to be the pore throat size, will be given by:

$$
\rho(b)=\rho_{i} \rho_{j}\left(\rho_{i}^{1 / n}+\rho_{j}^{1 / n}\right)^{-n}, \quad n>0,
$$

\section{References}

Acharya, R.C., van der Zee, S.E.A.T.M., Leijnse, A.: Porosity-permeability properties generated with a new 2-parameter 3D hydraulic pore-network model for consolidated and unconsolidated porous media. Adv. Water Resour. 27, 707-723 (2004)

Al-Raoush, R.I., Willson, C.S.: Extraction of physically realistic pore network properties from threedimensional synchrotron X-ray microtomography images of unconsolidated porous media systems. J. Hydrol. 300, 44-64 (2005a)

Al-Raoush, R.I., Willson, C.S.: A pore-scale investigation of a multiphase porous media system. J. Contam. Hydrol. 77, 67-89 (2005b)

Anwar, A.H.M.F., Bettahar, M., Matsubayashi, U.: A method for determining air-water interfacial area in variably saturated porous media. J. Contam. Hydrol. 43, 129-146 (2000)

Bear, J., Zaslavsky, D., Irmay, S.: Physical Principles of Water Percolation and Seepage. z.uitg, Paris (1968)

Blunt, M.J., Jackson, M.D., Piri, M., Valvatne, P.H.: Detailed physics, predictive capabilities and macroscopic consequences for pore-network models of multiphase flow. Adv. Water Resour. 25, 1069-1089 (2002)

Brusseau, M.L., Popovicova, J., Silva, J.A.K.: Characterizing gas-water interfacial and bulk-water partitioning for gas phase transport of organic contaminants in unsaturated porous media. Environ. Sci. Technol. 31, 1645-1649 (1997)

Brusseau, M.L., Peng, S., Schnaar, G., Constanza-Robinson, M.S.: Relationships among air-water interfacial area, capillary pressure, and water saturation for a sandy porous medium. Water Resour. Res. 42, W03501. doi: 10.1029/2005WR004058 (2006)

Burganos, V.N., Payatakes A., C.: Knudsen diffusion in random and correlated networks of constricted pores. Chem. Eng. Sci. 47, 1383-1400 (1992)

Chen, L., Kibbey, T.C.G.: Measurement of air-water interfacial area for multiple hysteretic drainage curves in an unsaturated fine sand. Langmuir 22, 6874-6880 (2006) 
Cheng, J.T., Pyrak-Nolte, L.J., Nolte, D.D.: Linking pressure and saturation through interfacial area in porous media, Geophys. Res. Lett. 31, L08502. doi: 10.1029/2003GL019282 (2004)

Costanza-Robinson, M.S., Brusseau, M.L.: Air-water interfacial areas in unsaturated soils: evaluation of interfacial domains. Water Resour. Res. 38, 13-1 (2002)

Culligan, K.A., Wildenschild, D., Christensen, B.S.B., Gray, W.G., Rivers, M.L., Tompson, A.F.B.: Interfacial area measurements for unsaturated flow through a porous medium. Water Resour. Res. 40, W12413. doi: 10.1029/2004WR003278 (2004)

Culligan, K.A., Wildenschild, D., Christensen, B.S.B., Gray, W., Rivers, M.L.: Pore-scale characteristics of multiphase flow in porous media: a comparison of air-water and oil-water experiments. Adv. Water Resour. 29, 227-238 (2006)

Dias, M.M., Payatakes, A.C.: Network models for two-phase flow in porous media, 2, motion of oil ganglia. J. Fluid Mech. 164, 337-358 (1986)

Fatt, I.: The network model of porous media. I. Capillary pressure characteristics. Pet. Trans. AIME 207, 144-159 (1956)

Gielen, T.W.J.: Upscaling Multiphase Transport Processes in Porous Media. Delft University of Technology (2007)

Hassanizadeh, S.M., Gray, W.G.: Mechanics and thermodynamics of multiphase flow in porous media including interface boundaries. Adv. Water Resour. 13, 169-186 (1990)

Hassanizadeh, S.M., Gray, W.G.: Thermodynamic basis of capillary pressure in porous media. Water Resour. Res. 29, 3389-3405 (1993a)

Hassanizadeh, S.M., Gray, W.G.: Toward an improved description of the physics of two-phase flow. Adv. Water Resour. 16, 53-67 (1993b)

Held, R.J., Celia, M.A.: Modeling support of functional relationships between capillary pressure, saturation, interfacial area and common lines. Adv. Water Resour. 24, 325-343 (2001)

Helland, J.O., Skjaeveland, S.M.: The relationship between capillary pressure, saturation and interfacial area from a model of mixed-wet triangular tubes. Water Resour. Res. 43, W12S10. doi:10.1029/ 2006WR005698 (2007)

Hui, M.H., Blunt, M.J.: Pore-scale modeling of three-phase flow and the effects of wettability, 2000SPE/DOE improved oil recovery symposium, Tulsa, Oklahoma, 3-5 April (2000)

Karkare, M.V., Fort, T.: Determination of the air-water interfacial area in wet "unsaturated" porous media. Langmuir 12, 2041-4044 (1996)

Kim, H., Rao, P.S.C., Annable, M.D.: Determination of effective air-water interfacial area in partially saturated porous media using surfactant adsorption. Water Resour. Res. 33, 2705-2711 (1997)

Kim, H., Rao, P.S.C., Annable, M.D.: Gaseous tracer technique for estimating air-water interfacial areas and interface mobility. Soil Sci. Soc. Am. J. 63, 1554-1560 (1999)

Mahmud, W.M., Nguyen, V.H.: Effects of snap-off in imbibition in porous media with different spatial correlations. Transp. Porous Med. 64, 279-300 (2006)

Montemagno, C.D., Gray, W.G.: Photoluminescent volumetric imaging: a technique for the exploration of multi-phase flow and transport in porous media. Geophys. Res. Lett. 22, 425-428 (1995)

Piri, M., Blunt, M.J.: Three-dimensional mixed-wet random pore-scale network modeling of two- and threephase flow in porous media, I. Model description. Phy. Rev. E71, 026301 (2005)

Reeves, P.C.: Development of porescale network model for the simulation of capillary pressure, saturation, interfacial area, relative permeability in multi-fluid porous media, PhD Thesis, Princeton University (1997)

Reeves, P.C., Celia, M.A.: A functional relationship between capillary pressure, saturation, and interfacial area as revealed by a pore-scale network model. Water Resour. Res. 32, 2345-2358 (1996)

Saripalli, K.P., Rao, P.S.C., Annable, M.D.: Determination of specific NAPL-water interfacial areas of residual NAPLs in porous media using the interfacial tracers technique. J. Contam. Hydrogeol. 30, 375-391 (1998)

Schaefer, C.E., Dicarlo, D.A., Blunt, M.J.: Experimental measurement of air-water interfacial area during gravity drainage and secondary imbibition in porous media. Water Resour. Res. 36, 885-890 (2000)

Schnaar, G., Brusseau, M.L.: Pore-scale characterization of organic immiscible-liquid morphology in natural porous media using synchrotron X-ray microtomography. Environ. Sci. Technol. 39, 8403-8410 (2005)

Schnaar, G., Brusseau, M.L.: Characterizing pore-scale configuration of organic immiscible liquid in multiphase systems with synchrotron X-ray microtomography. Vadose Zone J. 5, 641-648 (2006)

Vogel, H.J.: Morphological determination of pore connectivity as a function of pore size using serial sections. Eur. J. Soil Sci. 48, 365-377 (1997)

Vogel, H.J.: A numerical experiment on pore size, pore connectivity, water retention, permeability, and solute transport using network models. Eur. J. Soil Sci. 51, 99-105 (2000) 
Vogel, H.J., Roth, K.: A new approach for determining effective soil hydraulic functions. Eur. J. Soil Sci. 49, 547-556 (1998)

Wildenschild, D., Hopmans, J.W., Vaz, C.M.P., Rivers, M.L., Rikard, D.: Using X-ray computed tomography in hydrology: systems, resolutions, and limitations. J. Hydrol. 267, 285-297 (2002) 